\title{
A systematic classification of megakaryocytic dysplasia and its impact on prognosis for patients with myelodysplastic syndromes
}

Gege Feng ${ }^{1,2}$, Robert Peter Gale ${ }^{3}$, Wen Cui ${ }^{4}$, Wenyu Cai ${ }^{4}$, Gang Huang ${ }^{5}$, Zefeng Xu ${ }^{1,2}$, Tiejun Qin ${ }^{1}$, Yue Zhang ${ }^{1,2}$, Bing Li ${ }^{1,2}$, Liwei Fang ${ }^{1}$, Hongli Zhang ${ }^{1}$, Lijuan Pan ${ }^{1}$, Naibo Hu' ${ }^{1}$, Shiqiang Qu ${ }^{1}$, Jingya Wang ${ }^{2}$, Yajuan Cui ${ }^{2}$ and Zhijian Xiao ${ }^{1,2^{*}}$

\begin{abstract}
Background: Dys-megakaryopoiesis is defined as $\geq 10 \%$ of dysplastic megakaryocytes in bone marrow smears by the World Health Organization. However, concordance rates for dysplastic megakaryocytes between different observers is low and, consequently, evaluation of dysmegakaryopoiesis is also often discordant.

Results: We performed CD41 immune staining and proposed a systematic classification of dys-megakaryopoiesis on bone marrow films: (1) micro-megakaryocytes ( $<12 \mu \mathrm{m})$; (2) micro-megakaryocytes (12-40 $\mu \mathrm{m})$ with 1 nucleus; (3) micro-megakaryocytes (12-40 $\mu \mathrm{m}$ ) with 2 nuclei; (4) micro-megakaryocytes (12-40 um) with multiple (more than 2) nuclei; (5) dysplastic megakaryocytes ( $\geq 40 \mu \mathrm{m}$ ) with 1 nucleus; (6) dysplastic megakaryocytes ( $\geq 40 \mu \mathrm{m})$ with 2 nuclei; and (7) dysplastic megakaryocytes ( $\geq 40 \mu \mathrm{m}$ ) with multiple (more than 2 ) nuclei. Further, we evaluated the prognostic impact of micro-megakaryocytes and dysplastic mono-nucleated megakaryocytes on MDS patients. The best discriminator cut-off point for each group was determined by the minimal $P$ value approach. In multivariate analyses micro-megakaryocytes $\geq 25 \%$ and dysplastic mono-nucleated megakaryocytes $\geq 30 \%$ were independent adverse prognostic factors (hazard ratio $[\mathrm{HR}]=1.58$ [95 \% confidence interval $[\mathrm{Cl}], 1.11,2.23] ; \mathrm{P}=0.010$ and $1.53[1.09,2.16]$; $P=0.014)$.
\end{abstract}

Conclusions: Our data suggest integration of micro-megakaryocytes and dysplastic mono-nucleated megakaryocytes improve predictive accuracy of the international prognostic scoring system-revised (IPSS-R) scoring system.

Keywords: Dysplastic megakaryocytes, MDS, Immunochemistry, Prognosis

\section{Background}

Myelodysplastic syndromes (MDS) are a heterogeneous group of bone marrow neoplasms with variable clinical courses and prognoses [1]. Distinguishing the different form of MDS is important for accurate diagnosis, predicting outcomes and directing therapy. Several variables are used to distinguish different forms of MDS including

\footnotetext{
*Correspondence: zjxiao@hotmail.com

${ }^{1}$ MDS and MPN Centre, Institute of Hematology and Blood Diseases Hospital, Chinese Academy of Medical Sciences \& Peking Union Medical College, 288 Nanjing Road, Tianjin 300020, China

Full list of author information is available at the end of the article
}

morphology, histology, blood and bone marrow cell counts, cytogenetics and molecular genetics. Despite recent advances, cytological features in blood films and bone marrow aspirates and histological findings in trephine biopsies remain key elements for diagnosing MDS $[2,3]$. Among the histological parameters of MDS, multilineage dysplasia and percent bone marrow blasts are associated with unfavorable outcomes [4-7].

Megakaryocyte morphology is another important component in classifying MDS. The World Health Organization (WHO) 2008 classification defines dysmegakaryopoiesis as micro-megakaryocytes, hypo-lobed, 
or non-lobed nuclei in megakaryocytes of all sizes and multiple, widely-separated nuclei [8]. Although this definition of dys-megakaryopoiesis is potentially useful, there is no precise definition of micro-megakaryocytes in the WHO classification. Consequently it is not surprising that there is low concordance amongst observers for micro-megakaryocytes in bone marrow samples from persons with MDS [4, 9-12].

Megakaryocytes express surface CD41/CD61 and/or CD42b and CD42a $[13,14]$. The glycoprotein (Gp) IIb (CD41), which has been considered a specific marker for the megakaryocyte lineage [15], can be detected during megakaryocytic differentiation at a stage of a late megakaryocytic progenitor [16-18]. Consequently, using CD41 to identify megakaryocytes may be a better way to define dysplastic megakaryocytes than WrightGiemsa or May-Grünwald-Giemsa staining. We used CD41 immune staining to identify megakaryocytes and assess if they were dysplastic in bone marrow smears from persons with MDS. Further, we tried to describe the morphological features of megakaryocytic dysplasia by developing a systematic classification of megakaryocytic dysplasia and analyze the impact of our classification of dys-megakaryopoiesis on determining the prognosis of persons with MDS.

\section{Methods}

\section{Study cohort}

The study was approved by the ethics committees of the institute of hematology, Chinese Academy Of Medical Sciences (CAMS) and Peking Union Medical College (PUMC) according to guidelines of the declaration of Helsinki. In this retrospective analysis, the study cohort included 422 consecutive new-diagnosed subjects that were seen at the Institute of Hematology and Blood Disease Hospital, Chinese Academy of Medical Sciences from January, 2000 to April, 2014. 8 subsequently received a haematopoietic cell transplant, 14, decitabine, 45 , other chemotherapy and the remainder cyclosporine or thalidomide and best supportive care. Cases were re-reviewed by two blinded pathologists (W Cui and W Cai) and classified using the 2008 WHO criteria [2]. Subjects with suspected therapy-related MDS were excluded as the clinical course was typically progressive and treatment with conventional therapy was usually associated with a poor prognosis [19]. Furthermore, there was no Down Syndrome patient in the cohort. Follow-up data were available for 370 subjects $(88 \%)$. Date of last follow-up was December 15, 2014 or date of last contact. Median follow-up was 22 months (range 1-180 months). Subjects with lower-risk MDS fall into the international prognostic scoring system-revised (IPSS-R) categories of very low-, low-, and intermediate-risk groups and those with higher-risk MDS into the high- and very high-risk groups [20].

\section{Cytologic analysis}

Bone marrow smears from diagnosis were reviewed using an avidin-biotin-complex method (ABC; CD41 immune staining) by the experts who were blinded for patients' diagnoses, cytopenias and cytogenetic status in cytology. The preparation of bone marrow smear was a relatively uniform procedure. The marrow area on every smear was approximate to $1.5 \times 3.0 \mathrm{~cm}$ with proper and relatively uniform thickness. $\geq 30$ megakaryocytes were evaluated and the frequency of morphologic abnormalities was recorded. The presence of nuclear hypolobation, single or multiple separate small round nuclei were considered as main characteristics of dys-megakaryocytopoiesis.

\section{Statistical analyses}

Statistical analyses were performed using SPSS 19.0 software or SAS software. The best discriminator threshold was detected using the minimal $P$ value approach (a method aimed at minimizing the identification of rare classes of subjects) and considering survival (log-rank statistic) as the dependent variable [21, 22]. The functional form of the covariate under study was also evaluated using Martingale residual analysis [23].

Numerical variables were summarized by median and range. Categorical variables were described with count and relative frequency (\%) of subjects in each category. Comparison of numerical variables between groups was carried out using a non-parametric approach (MannWhitney test). Comparison of the distribution of categorical variables in different groups was performed with $\chi^{2}$ test (unordered categorical variable) or the non-parametric approach (ordinal categorical variable).

Median survival was estimated using the KaplanMeier method and compared using the log-rank test. Cox proportional hazard regression model was used for multivariate analyses. $P$ values were two-tailed and statistical significance was set as the level of $\mathrm{P}<0.05$.

\section{Results}

\section{Subjects variables}

Median age at diagnosis was 50 years (range $16-83$ years). 286 subjects ( $68 \%$ ) were male. Distribution of WHO subtypes, IPSS-R cytogenetic category and IPSS-R classification are indicated in Table 1.

Cyto-morphologic evaluation of megakaryocyte dysplasia Megakaryocyte dysplasia was detected in 374 subjects (89 \%). Median frequency of dysplastic megakaryocytes was $14 \%$ (range $0-100 \%$ ). Patients without megakaryocytic dysplasia included RA (17 \%), RARS (15\%), 
Table 1 Clinical variables $(\mathrm{N}=\mathbf{4 2 2})$

\begin{tabular}{ll}
\hline & Subjects $(\mathbf{n}=\mathbf{4 2 2})$ \\
\hline Median age (range, years) & $50(16-83)$ \\
Male & $286(68 \%)$ \\
WHO classification & \\
RA & $22(5 \%)$ \\
RN & 2 \\
RT & 3 \\
RARS & $24(6 \%)$ \\
RCMD & $198(47 \%)$ \\
RAEB-1 & $84(20 \%)$ \\
RAEB-2 & $76(18 \%)$ \\
MDS-U & 8 \\
MDS with del(5q) only & 5 \\
IPSS-R cytogenetic category & \\
Very-good & 6 \\
Good & $208(49 \%)$ \\
Intermediate & $143(34 \%)$ \\
Poor & $23(5 \%)$ \\
Very-poor & $42(10 \%)$ \\
IPSS-R (\%) & \\
Very-low & 6 \\
Low & $99(23 \%)$ \\
Intermediate & $135(32 \%)$ \\
High & $105(25 \%)$ \\
Very-high & $77(18 \%)$ \\
\hline
\end{tabular}

RCMD (42 \%), RAEB-1 (17 \%), RAEB-2 (6 \%), MDS-U (4\%). Dysplastic megakaryocytes were assigned to 7 categories: (1) micro-megakaryocytes $(<12 \mu \mathrm{m})$; (2) micro-megakaryocytes $(12-40 \mu \mathrm{m})$ with 1 nucleus; (3) micro-megakaryocytes $(12-40 \mu \mathrm{m})$ with 2 nuclei; (4) micro-megakaryocytes (12-40 um) with multiple (more than 2) nuclei; (5) dysplastic megakaryocytes $(\geq 40 \mu \mathrm{m})$ with 1 nucleus; (6) dysplastic megakaryocytes $(\geq 40 \mu \mathrm{m})$ with 2 nuclei; and (7) dysplastic megakaryocytes $(\geq 40 \mu \mathrm{m}$ ) with multiple (more than 2 ) nuclei (Fig. 1 ). The most frequent dysplastic megakaryocytes were micromegakaryocytes $(12-40 \mu \mathrm{m})$ with 1 nucleus and dysplastic megakaryocytes $(\geq 40 \mu \mathrm{m})$ with 1 nucleus, with median frequency of $28 \%(0-91 \%)$ and $29 \%(0-78 \%)$, respectively. Distribution of each type is shown in Additional file 1: Figure S1.

To analyze the prognostic impact of dys-megakaryopoiesis, we identified the cutoff point for the two prognostic classes with the greatest differences according to the smallest P value for micro-megakaryocytes at $25 \%$ as well as mono-nucleated dys-megakaryopoiesis at $30 \%$. Subjects without megakaryocytic dysplasia were all grouped to micro-megakaryocytes $<25 \%$ and mononucleated dys-megakaryopoiesis $<30 \%$.
Association between subject variables and megakaryocyte dysplasia

Clinical and laboratory variables in subjects with micromegakaryocytes $<25$ and $\geq 25 \%$ are compared in Additional file 1: Table S1. A similar comparison between subjects with mono-nucleated dys-megakaryopoiesis $<30$ and $\geq 30 \%$ is outlined in Additional file 1: Table S2.

Increased micro-megakaryocytes and mono-nucleated dys-megakaryopoiesis were significantly associated with lower levels of platelet count $(\mathrm{P}<0.001$ and $\mathrm{P}<0.001)$ and higher levels of bone marrow blasts $(\mathrm{P}<0.001$ and $\mathrm{P}<0.001)$. Distributions of WHO 2008 subtypes $(\mathrm{P}=0.001$ and $\mathrm{P}<0.001)$, IPSS-R cytogenetic category $(\mathrm{P}=0.002$ and $\mathrm{P}=0.001)$ and IPSS-R risk cohorts $(\mathrm{P}<0.001$ and $\mathrm{P}<0.001)$ were also significantly different. There was no significant difference in age, gender, hemoglobin concentration and blood neutrophil counts at diagnosis between the two groups. In addition, levels of micro-megakaryocytes and dysplastic mono-nucleated megakaryocytes were significantly associated with abnormal karyotype $(\mathrm{P}=0.026$ and $\mathrm{P}=0.014)$, complex karyotype $(\mathrm{CK})(\mathrm{P}=0.034$ and $\mathrm{P}=0.022)$ and chromosome 7 aberrations $(P=0.004$ and $P=0.003)$, but not with monosomal karyotype (MK) or $\operatorname{del}(5 \mathrm{q})$ (Additional file 1: Table S3).

\section{Prognostic implications of megakaryocyte dysplasia}

In univariate analyses, subjects with micro-megakaryocytes $\geq 25 \%$ had poorer survival (median, 19 months [95 \% CI 14-23 months]) than those with micromegakaryocytes $<25 \%$ (46 months [28-64 months], $\mathrm{P}<0.001$; Fig. 2a). Similarly, patients with dysplastic mono-nucleated megakaryocytes $\geq 30 \%$ demonstrated poorer survival as compared to those with dysplastic mono-nucleated megakaryocytes $<30 \%$ (18 months [14-23 months] vs. 49 months [31-68 months], $\mathrm{P}<0.001$; Fig. 2b). Other significant predictors of survival in univariate analyses were male gender $(\mathrm{P}=0.009)$, age $\geq 60$ years $(\mathrm{P}<0.001)$, hemoglobin concentration $<80 \mathrm{~g} / \mathrm{L}(\mathrm{P}=0.001)$, neutrophils $<0.8 \times 10 \mathrm{E}+9 / \mathrm{L}$ $(\mathrm{P}=0.001)$, platelets $<50 \times 10 \mathrm{E}+9 / \mathrm{L}(\mathrm{P}=0.002)$, bone marrow blasts $>10 \%(\mathrm{P}<0.001)$, IPSS-R cytogenetic category $(\mathrm{P}<0.001)$ and IPSS-R score $(\mathrm{P}<0.001)$.

We performed a multivariate Cox regression model including gender, age, micro-megakaryocytes and IPSS-R. Male gender (hazard ratio $[\mathrm{HR}]=1.5$; $95 \% \mathrm{CI}$ $1.0-2.0 ; \mathrm{P}=0.029)$, age $\geq 60$ years $(\mathrm{HR}=1.5 ;[1.1-2.0]$; $\mathrm{P}=0.016)$, micro-megakaryocytes $\geq 25 \%(\mathrm{HR}=1.6$ [1.1-2.2]; $\mathrm{P}=0.010)$ and IPSS- $\mathrm{R}$ score $(\mathrm{P}<0.001)$ were significantly associated with survival (Table 2). In a similar analysis including gender, age, dysplastic mononucleated megakaryocytes and IPSS-R, only male gender $(\mathrm{HR}=1.5 ; 95 \%$ CI 1.0-2.0; $\mathrm{P}=0.028)$, age $\geq 60$ years 

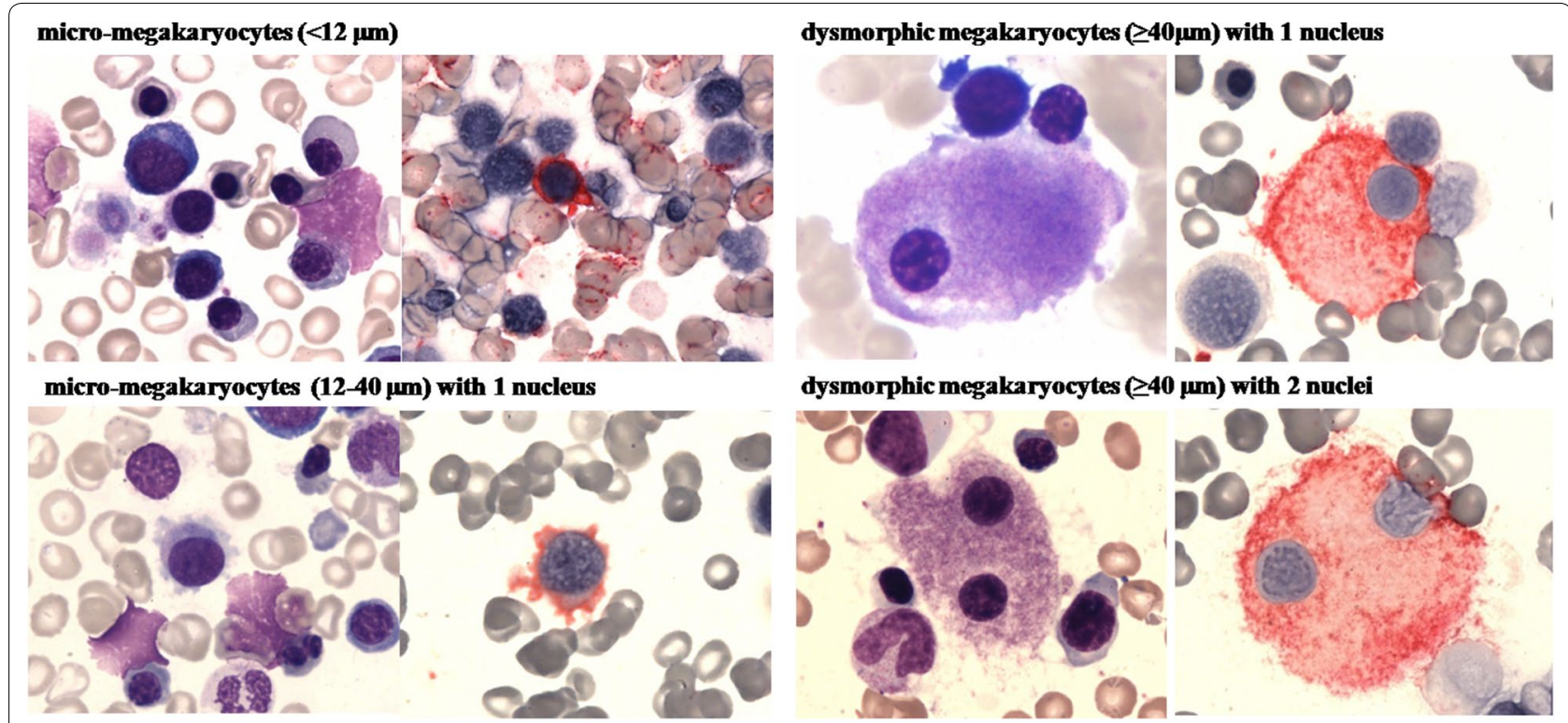

dysmorphic megakaryocytes $(\geq 40 \mu \mathrm{m})$ with 2 nuclei
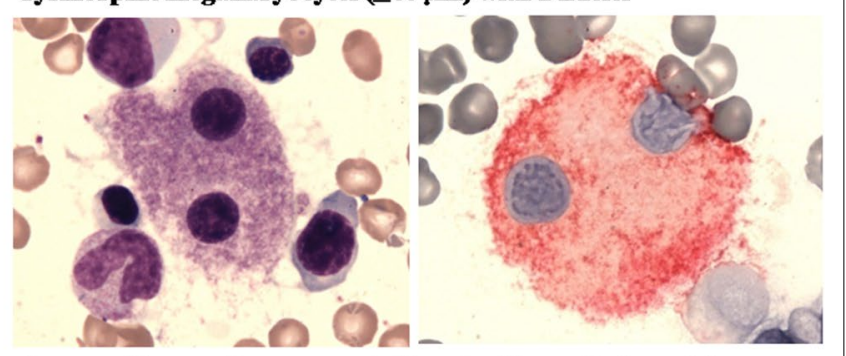

micro-megakaryocytes (12-40 $\mu \mathrm{m})$ with 2 nuclei

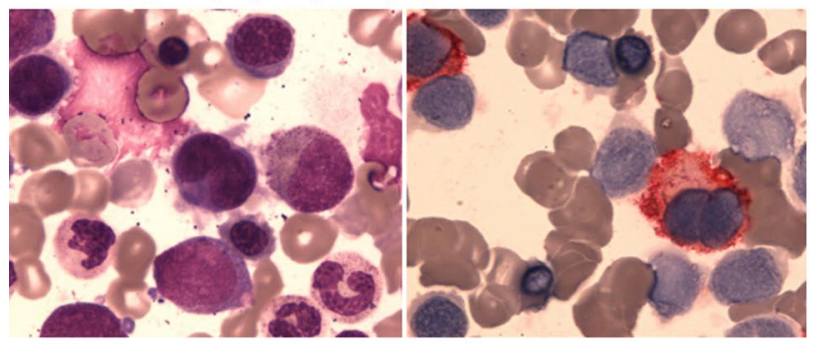

dysmorphic megakaryocytes $(\mathbb{4 0} \mu \mathrm{m})$ with multiple nuclei
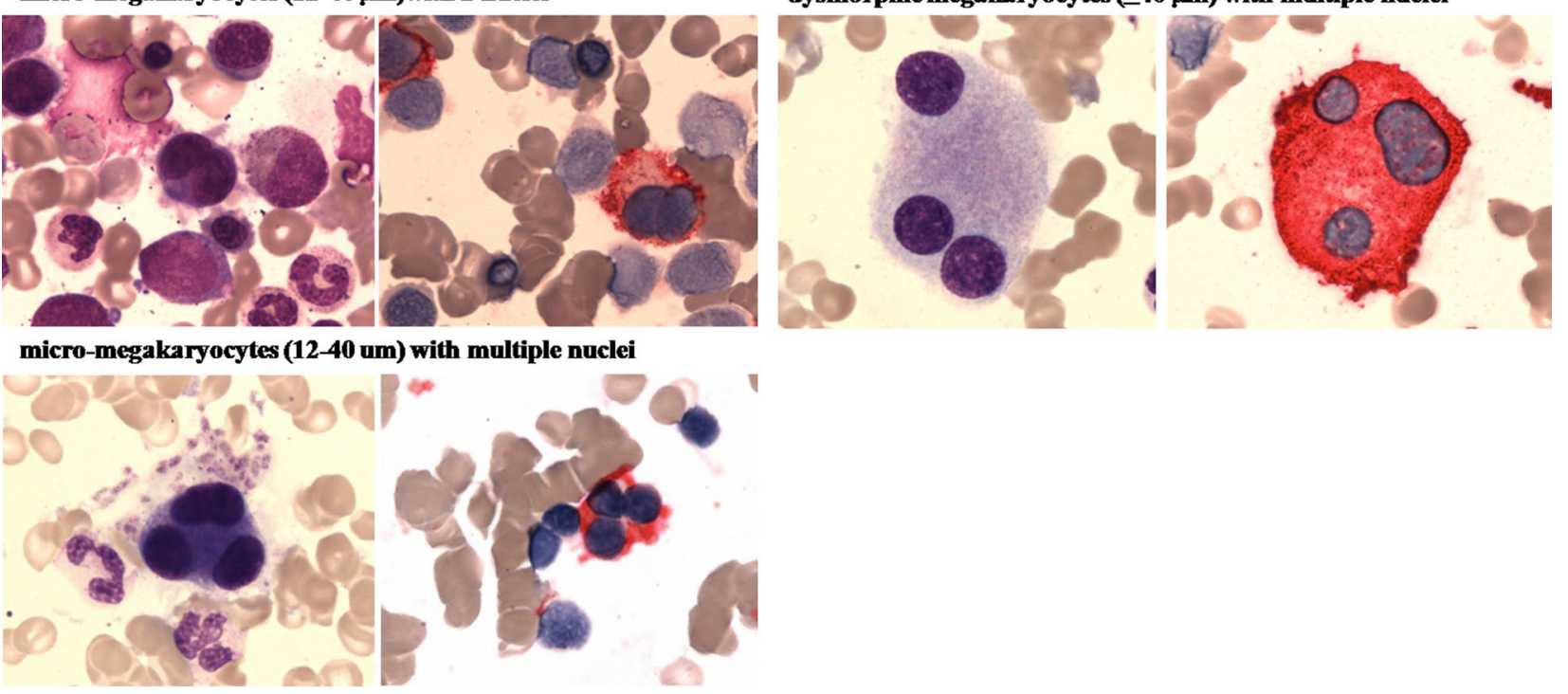

Fig. 1 Wright-Giemsa staining and CD41 immune staining of dysplastic megakaryocytes on bone marrow smears

$(\mathrm{HR}=1.5 ; 95 \%$ CI 1.1-2.0; $\mathrm{P}=0.018)$, dysplastic mononucleated megakaryocytes $\geq 30 \%(\mathrm{HR}=1.533 ;[1.1-2.2]$; $\mathrm{P}=0.014)$ and IPSS-R $(\mathrm{P}<0.001)$ remained in the final model (Table 3).

\section{Prognostic implications of megakaryocytic dysplasia} in IPSS-R lower-risk subjects according to the IPSS-R score In the IPSS-R lower-risk subjects, those with micromegakaryocytes $\geq 25 \%$ had a poorer survival than patients with micro-megakaryocytes $<25 \%(\mathrm{P}<0.001$; Fig. 3a). Similarly, there was a significant difference in survival between patients with mono-nucleated dys-megakaryopoiesis $<30$ and $\geq 30 \%$ ( $\mathrm{P}<0.001$; Fig. $3 \mathrm{~b})$. This association was not significant in subjects in the IPSS-R higher-risk cohort. Consequently, we performed stratified multivariate analyses to further evaluate prognostic implications of megakaryocytic dysplasia in IPSS-R lower-risk subjects only. In the model of cell size including age, gender, IPSS-R and micro-megakaryocytes, only age, IPSS-R and micro-megakaryocytes were significantly correlated with survival (Table 4). In a similar analysis considering dysplastic mono-nucleated megakaryocytes, only age, IPSS-R and dysplastic mono-nucleated megakaryocytes were significantly correlated with survival (Table 5). 

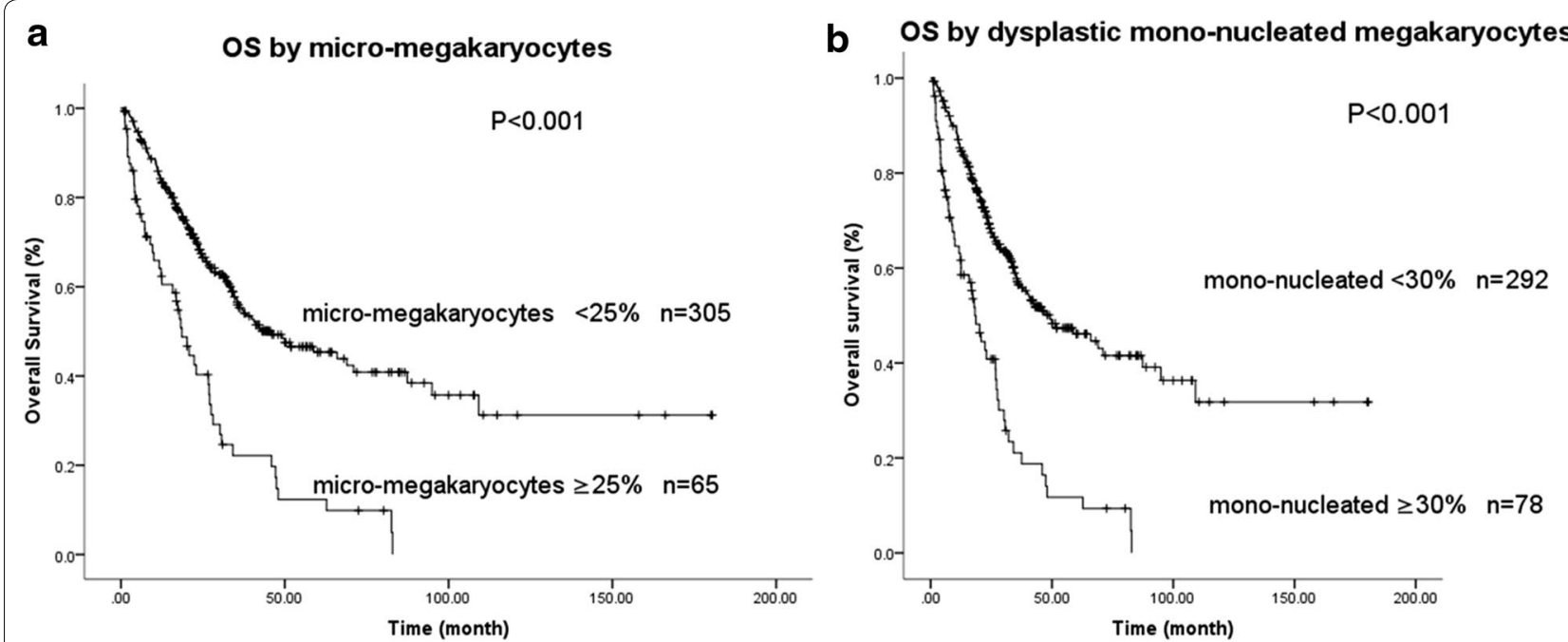

Fig. 2 a Survival of 370 subjects with micro-megakaryocyte $<25$ or $\geq 25 \%$; b Survival of subjects with dysplastic mono-nucleated megakaryocytes $<30$ or $\geq 30 \%$

Table 2 Multivariate Cox regression analysis with respect to micro-megakaryocytes

\begin{tabular}{|c|c|c|c|}
\hline & HR & $95 \% \mathrm{Cl}$ & $P$ value \\
\hline Gender & & & 0.029 \\
\hline Male & 1.458 & $1.039-2.047$ & \\
\hline Female* & 1.0 & & \\
\hline Age (year) & & & 0.016 \\
\hline$\geq 60$ & 1.485 & $1.078-2.047$ & \\
\hline$<60^{*}$ & 1.0 & & \\
\hline IPSS-R & & & $<0.001$ \\
\hline Very-low & 0 & $0-2.31 E 133$ & \\
\hline Low & 0.106 & $0.062-0.180$ & \\
\hline Intermediate & 0.227 & $0.151-0.341$ & \\
\hline High & 0.597 & $0.410-0.869$ & \\
\hline Very-high* & 1.0 & & \\
\hline Micro-megakaryocytes (\%) & & & 0.010 \\
\hline$\geq 25$ & 1.575 & $1.113-2.230$ & \\
\hline$<25^{*}$ & 1.0 & 1.0 & \\
\hline
\end{tabular}

* The reference

\section{Discussion}

In the WHO classification of myeloid neoplasms bone marrow dysplasia $\geq 10 \%$ of the cells of a specific myeloid lineage is the cardinal diagnostic feature of the MDS. However, morphology is a subjective parameter under the routine staining conditions. For example, concordance for dys-megakaryopoiesis amongst observers was less than concordance for dys-granulopoiesis and dyserythropoiesis [4]. Thus, specific immune staining may be a better way to evaluate megakaryocytes than routine histological methods [24].
Table 3 Multivariate Cox regression analysis with respect to dysplastic mono-nucleated megakaryocytes

\begin{tabular}{|c|c|c|c|}
\hline & HR & $95 \% \mathrm{Cl}$ & P value \\
\hline Gender & & & 0.028 \\
\hline Male & 1.465 & $1.042-2.058$ & \\
\hline Female* & 1.0 & & \\
\hline Age (year) & & & 0.018 \\
\hline$\geq 60$ & 1.474 & $1.068-2.034$ & \\
\hline$<60^{*}$ & 1.0 & - & \\
\hline IPSS-R & & & $<0.001$ \\
\hline Very-low & 0 & $0-5.24 \mathrm{E} 132$ & \\
\hline Low & 0.108 & $0.063-0.185$ & \\
\hline Intermediate & 0.235 & $0.155-0.355$ & \\
\hline High & 0.607 & $0.416-0.885$ & \\
\hline Very-high* & 1.0 & & \\
\hline Mono-nucleated (\%) & & & 0.014 \\
\hline$\geq 30$ & 1.533 & $1.089-2.160$ & \\
\hline$<30^{*}$ & 1.0 & & \\
\hline
\end{tabular}

*The reference

Based on data from our study we propose a systematic classification of dys megakaryopoiesis on bone marrow films of persons with suspected MDS using CD41 immune staining. A study has reviewed marrow smears of 26 RA and 28 RAEB patients, and micro-megakaryocytes were identified in 39.3 and $46.5 \%$ cases under Wright-Giemsa staining, compared with 92.3 and $100 \%$ under CD41 immune staining ( $\mathrm{X} 2$ test: $\mathrm{P}<0.005$ and $\mathrm{P}=0.01$ ) (Wenyu Cai, unpublished data).

In our study, a larger number of dysplastic megakaryocytes was significantly associated with decreased platelet 


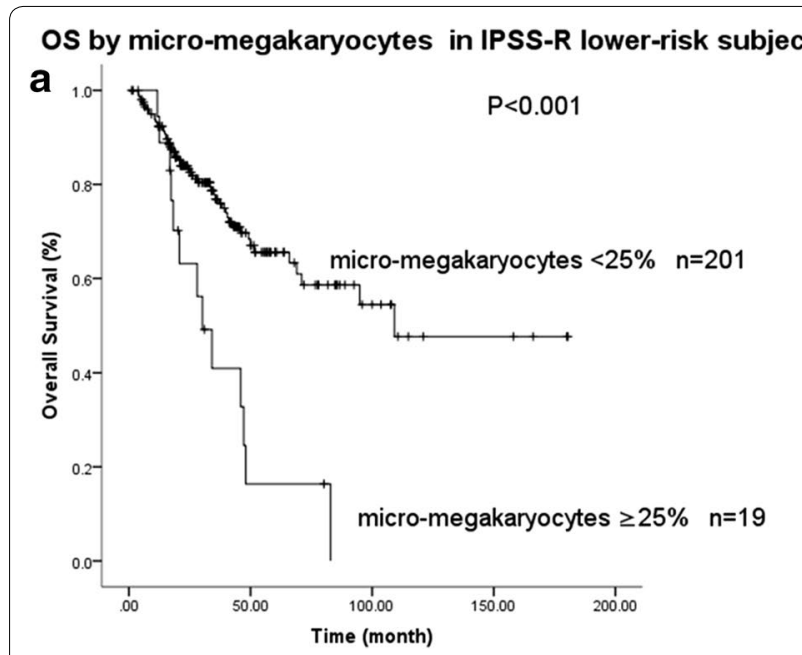

OS by dysplastic mono-nucleated megakaryocytes in IPSS-R

b lower-risk subjects

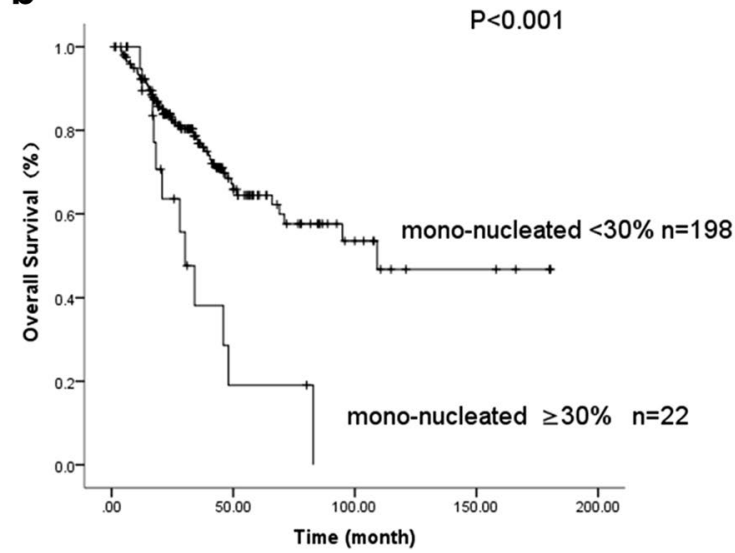

Fig. 3 a Survival of subjects with IPSS-R lower-risk with micro-megakaryocyte $<25$ or $\geq 25 \%$; b survival of subjects with dysplastic mono-nucleated megakaryocytes $<30$ or $\geq 30 \%$

Table 4 Multivariate analysis of the survival of IPSS-R lower-risk subjects with respect to micro-megakaryocytes

\begin{tabular}{lllr}
\hline & HR & $\mathbf{9 5} \% \mathbf{C l}$ & P value \\
\hline $\begin{array}{lll}\text { Age (year) } \\
\geq 60\end{array}$ & 1.856 & $1.086-3.171$ & \\
$\quad<60^{*}$ & 1.0 & & \\
Gender & & & 0.024 \\
$\quad$ IPSS-R & & & 0.026 \\
$\quad$ Very-low & 0 & $0-6.17 \mathrm{E} 272$ & \\
Low & 0.478 & $0.280-0.816$ & \\
Intermediate* & 1.0 & & \\
Micro-megakaryocytes (\%) & & & \\
$\geq 25$ & 2.806 & $1.509-5.218$ & \\
$<25^{*}$ & 1.0 & & \\
\hline
\end{tabular}

*The reference

count, increased bone marrow blasts and increased IPSS$\mathrm{R}$ scores. Cytogenetic abnormalities are associated with characteristic dysplastic features, e.g. isolated $\operatorname{del}(5 \mathrm{q})$ and hypo-lobed and un-lobed megakaryocyte nuclei and del(17p) with hypo-lobed neutrophil nuclei [25]. Data from our study also indicated parallel increases in micro-megakaryocytes and dysplastic mono-nucleated megakaryocytes and IPSS-R cytogenetic scores, as well as frequencies of abnormal karyotype, $\mathrm{CK}$ and chromosome 7 aberrations. However, according to our study, there was no significant association of dysplastic megakaryocytes with del(5q). Hypolobulation is also commonly seen in all other subtypes besides MDS with $\operatorname{del}(5 \mathrm{q})$ only. This unexpected result may be on account of the small proportion of $\operatorname{del}(5 \mathrm{q})$ patients in our study cohort (5/422). A larger
Table 5 Multivariate analysis of the survival of IPSS-R lower-risk subjects with respect to dysplastic mono-nucleated megakaryocytes

\begin{tabular}{lllc}
\hline & HR & $95 \% \mathbf{C l}$ & P value \\
\hline $\begin{array}{lll}\text { Age (year) } \\
\geq 60\end{array}$ & 1.808 & $1.058-3.089$ & 0.03 \\
$<60^{*}$ & 1.0 & & \\
$\begin{array}{l}\text { Gender } \\
\text { IPSS-R }\end{array}$ & & & 0.161 \\
Very-low & 0 & $0-1.07 E 266$ & 0.018 \\
Low & 0.463 & $0.272-0.789$ & \\
Intermediate* & 1.0 & & $<0.01$ \\
Mono-nucleated (\%) & & & \\
$\geq 30$ & 2.672 & $1.410-5.064$ & \\
$<30^{*}$ & 1.0 & & \\
\hline * The reference & & & \\
\end{tabular}

dataset is necessary for further research on this issue. Furthermore, we suspect that abnormalities in process of endo-reduplication may play a more important role than aberrant endomitosis in dysplastic megakaryocytes, for micro-megakaryocytes with 1 nucleus and dysplastic megakaryocytes $(\geq 40 \mu \mathrm{m})$ with 1 nucleus were most frequently seen in our study.

Studies reported a threshold of $10 \%$ dysplastic granulocytes as well as 30-40 \% dysplastic megakaryocytes as the best survival discriminator [4, 11, 26, 27]. We found a threshold of $25 \%$ of micro-megakaryocytes and $30 \%$ of dysplastic mono-nucleated megakaryocytes as the best survival discriminators, which was independent of age, gender and IPSS-R risk score. We also found 
micro-megakaryocytes and dysplastic mono-nucleated megakaryocytes were independently associated with survival in subjects with IPSS-R lower-risk MDS. Based on these data we suggest that adding these variables to the IPSS- $\mathrm{R}$ model could improve the predictive accuracy in untreated subjects and those with IPSS-R lowerrisk MDS. External validation of concordance between observers using our technique and dysplastic megakaryocytes classification is needed.

\section{Additional file}

Additional file 1. Distribution of dysplastic megakaryocytes in subjects and variables of subjects with dysplastic megakaryocytes.

\section{Authors' contributions}

ZJX designed the research, was the principal investigator, and took primary responsibility for the paper; GF acquisition of data, analysis and interpretation of data, and drafting the article; WC, WC, GH acquisition of data; TJQ, YZ, LWF, HLZ, LJP, NBH, SQQ, BL and ZJX recruited the patients; ZJX, RPG, $J M B, P L G$ wrote the typescript. All authors read and approved the final manuscript.

\section{Author details \\ ${ }^{1}$ MDS and MPN Centre, Institute of Hematology and Blood Diseases Hospital, Chinese Academy of Medical Sciences \& Peking Union Medical College, 288 Nanjing Road, Tianjin 300020, China. ${ }^{2}$ State Key Laboratory of Experimental Hematology, Institute of Hematology and Blood Diseases Hospital, Chinese Academy of Medical Sciences \& Peking Union Medical College, Tianjin, China. ${ }^{3}$ Hematology Research Center, Division of Experimental Medicine, Depart- ment of Medicine, Imperial College London, London, UK. ${ }^{4}$ Department of Pathology, Institute of Hematology and Blood Diseases Hospital, Chinese Academy of Medical Sciences \& Peking Union Medical College, Tianjin, China. ${ }^{5}$ Divisions of Experimental Hematology and Cancer Biology, Cincinnati Chil- dren's Hospital Medical Center, Cincinnati, OH, USA.}

\section{Acknowledgements}

Supported in part by National Natural Science Funds (No. 81470295, No. 81470297, No. 81370611, No. 81530008), Program for Peking Union Scholars and Innovative Research Team and National Key Technology R\&D Program (No. 2014BAI09B13). RPG acknowledges support from the NIHR Biomedical Research Centre funding scheme. We thanks Professor B. Bain (London, UK) for her very helpful suggestions.

\section{Competing interests}

RPG is a part-time employee of Celgene Corp. The authors declare that they have no competing interests.

Received: 4 April 2016 Accepted: 8 April 2016

Published online: 27 April 2016

\section{References}

1. Tefferi A, Vardiman JW. Myelodysplastic syndromes. N Engl J Med. 2009;361:1872-85.

2. Vardiman JW, Thiele J, Arber DA, Brunning RD, Borowitz MJ, Porwit A, et al. The 2008 revision of the World Health Organization (WHO) classification of myeloid neoplasms and acute leukemia: rationale and important changes. Blood. 2009;114:937-51.
3. Malcovati L, Hellstrom-Lindberg E, Bowen D, Ades L, Cermak J, Del Canizo $\mathrm{C}$, et al. Diagnosis and treatment of primary myelodysplastic syndromes in adults: recommendations from the European LeukemiaNet. Blood. 2013;122:2943-64.

4. Matsuda A, Germing U, Jinnai I, Iwanaga M, Misumi M, Kuendgen A et al. Improvement of criteria for refractory cytopenia with multilineage dysplasia according to the WHO classification based on prognostic significance of morphological features in patients with refractory anemia according to the FAB classification. Leukemia. 2007;21:678-86.

5. Greenberg P, Cox C, LeBeau MM, Fenaux P, Morel P, Sanz G, et al. International scoring system for evaluating prognosis in myelodysplastic syndromes. Blood. 1997;89:2079-88.

6. Malcovati L, Germing U, Kuendgen A, Della Porta MG, Pascutto C, Invernizzi R, et al. Time-dependent prognostic scoring system for predicting survival and leukemic evolution in myelodysplastic syndromes. J Clin Oncol. 2007;25:3503-10.

7. Greenberg PL, Tuechler H, Schanz J, Sanz G, Garcia-Manero G, Sole F, et al. Revised international prognostic scoring system for myelodysplastic syndromes. Blood. 2012;120:2454-65.

8. Swerdllow S, Campo E, Harris NL. WHO classification of tumours of haematopoietic and lymphoid tissues. France: IARC Press; 2008. p. 2008.

9. Kuriyama K, Tomonaga M, Matsuo T, Ginnai I, Ichimaru M. Diagnostic significance of detecting pseudo-Pelger-Huet anomalies and micro-megakaryocytes in myelodysplastic syndrome. Br J Haematol. 1986;63:665-9.

10. Matsuda A, Jinnai I, Yagasaki F, Kusumoto S, Minamihisamatsu M, Honda $\mathrm{S}$, et al. Refractory anemia with severe dysplasia: clinical significance of morphological features in refractory anemia. Leukemia. 1998;12:482-5.

11. Della Porta MG, Travaglino E, Boveri E, Ponzoni M, Malcovati L, Papaemmanuil $E$, et al. Minimal morphological criteria for defining bone marrow dysplasia: a basis for clinical implementation of WHO classification of myelodysplastic syndromes. Leukemia. 2015;29:66-75.

12. Germing U, Strupp C, Giagounidis A, Haas R, Gattermann N, Starke C, et al. Evaluation of dysplasia through detailed cytomorphology in 3156 patients from the Dusseldorf Registry on myelodysplastic syndromes. Leukemia Res. 2012;36:727-34.

13. Abgrall JF, Berthou C, Sensebe L, Le Niger C, Escoffre M. Decreased in vitro megakaryocyte colony formation in chronic idiopathic thrombocytopenic purpura. Br J Haematol. 1993;85:803-4.

14. Alimardani G, Guichard J, Fichelson S, Cramer EM. Pathogenic effects of anti-glycoprotein $\mathrm{lb}$ antibodies on megakaryocytes and platelets. Thromb Haemost. 2002;88:1039-46.

15. Vinci G, Tabilio A, Deschamps JF, Van Haeke D, Henri A, Guichard J, et al. Immunological study of in vitro maturation of human megakaryocytes. Br J Haematol. 1984:56:589-605.

16. Rabellino EM, Levene RB, Leung LL, Nachman RL. Human megakaryocytes. II. Expression of platelet proteins in early marrow megakaryocytes. J Exp Med. 1981;154:88-100.

17. Kanz L, Mielke R, Fauser AA. Analysis of human hemopoietic progenitor cells for the expression of glycoprotein Illa. Exp Hematol. 1988;16:741-7.

18. Debili N, Issaad C, Masse JM, Guichard J, Katz A, Breton-Gorius J, et al. Expression of CD34 and platelet glycoproteins during human megakaryocytic differentiation. Blood. 1992:80:3022-35.

19. Bhatia S. Therapy-related myelodysplasia and acute myeloid leukemia. Semin Oncol. 2013;40:666-75

20. Sekeres MA, Gerds AT. Established and novel agents for myelodysplastic syndromes. Hematol Am Soc Hematol Educ Progr. 2014;2014:82-9.

21. Lausen B, Schumacher M. Maximally selected rank statistics. Biometrics. 1992: 73-85.

22. Altman DG, Lausen B, Sauerbrei W, Schumacher M. Dangers of using "optimal" cutpoints in the evaluation of prognostic factors. J Natl Cancer Inst. 1994;86:829-35.

23. Therneau TM, Grambsch PM, Fleming TR. Martingale-based residuals for survival models. Biometrika. 1990;77:147-60.

24. Das R, Hayer J, Dey P, Garewal G. Comparative study of myelodysplastic syndromes and normal bone marrow biopsies with conventional staining and immunocytochemistry. Anal Quant Cytol Histol. $2005 ; 27: 152-6$ 
25. Lai JL, Preudhomme C, Zandecki M, Flactif M, Vanrumbeke M, Lepelley $\mathrm{P}$, et al. Myelodysplastic syndromes and acute myeloid leukemia with $17 p$ deletion. An entity characterized by specific dysgranulopoiesis and a high incidence of P53 mutations. Leukemia. 1995;9:370-81.

26. Germing U, Gattermann N, Strupp C, Aivado M, Aul C. Validation of the WHO proposals for a new classification of primary myelodysplastic syndromes: a retrospective analysis of 1600 patients. Leukemia Res. 2000;24:983-92.
27. Matsuda A, Germing U, Jinnai I, Misumi M, Kuendgen A, Knipp S, et al. Difference in clinical features between Japanese and German patients with refractory anemia in myelodysplastic syndromes. Blood. 2005;106:2633-40.

\section{Submit your next manuscript to BioMed Central and we will help you at every step:}

- We accept pre-submission inquiries

- Our selector tool helps you to find the most relevant journal

- We provide round the clock customer support

- Convenient online submission

- Thorough peer review

- Inclusion in PubMed and all major indexing services

- Maximum visibility for your research

Submit your manuscript at

www.biomedcentral.com/submit 